\title{
OR10-004 - Circulating micrornas in TRAPS
}

\author{
OM Lucherini ${ }^{*}$, L Obici ${ }^{2}$, M Ferracin ${ }^{3}$, V Fulci ${ }^{4}$, MF McDermott ${ }^{5}$, G Merlini ${ }^{6}$, I Muscari ${ }^{7}$, F Magnotti ${ }^{1}$, LJ Dickie $^{5}$, \\ M Galeazzi ${ }^{7}$, M Negrini $^{3}$, CT Baldari ${ }^{8}$, R Cimaz $^{9}$, L Cantarini $^{10}$ \\ From 7th Congress of International Society of Systemic Auto-Inflammatory Diseases (ISSAID) \\ Lausanne, Switerland. 22-26 May 2013
}

\section{Introduction}

To the best of our knowledge circulating miRNAs in TRAPS, as well as in other monogenic autoinflammatory disorders have never been investigated.

\section{Objectives}

To evaluate circulating microRNAs (miRNAs) levels in patients with tumor necrosis factor-receptor associated periodic syndrome (TRAPS), in comparison to healthy controls, and to correlate their levels to parameters of disease activity and/or disease severity.

\section{Methods}

Expression levels of circulating miRNAs were measured by Agilent microarrays in 29 serum samples from 15 TRAPS patients carrying mutations known to be associated with high disease penetrance and 8 healthy controls. Differentially expressed and clinically relevant miRNAs were detected using GeneSpring GX software.

\section{Results}

We identified a 6 miRNAs signature able to discriminate TRAPS from healthy controls. Moreover, 4 miRNAs were differentially expressed between patients treated with the interleukin (IL)-1 receptor antagonist anakinra and untreated patients. Of these, miR-92a-3p expression was found to be reduced in untreated patients, while its expression levels were similar to healthy controls in samples obtained during anakinra treatment. MiR-92b levels were inversely correlated with the number of fever attacks/year during the $1^{\text {st }}$ year from the index attack of TRAPS, while miR-377-5p levels were positively correlated with serum amyloid A (SAA) circulating levels.

\section{Conclusion}

Serum miRNAs levels show a baseline pattern in TRAPS, and may serve as potential markers of response to therapeutic intervention.

\section{Competing interests}

None declared.

\section{Authors' details}

${ }^{1}$ Department of Medical Sciences, Surgical and Neuroscience. Rheumatology Unit, Università di Siena, Siena, Italy. ${ }^{2}$ Amyloid Research and Treatment Center, Fondazione IRCCS Policlinico San Matteo, and Department of Molecular Medicine., University of Pavia, Pavia, Italy. ${ }^{3}$ Laboratory for Technologies of Advanced Therapies (LTTA) and Department of Morphology, Surgery and Experimental Medicine., University of Ferrara, Ferrara, Italy.

${ }^{4}$ Dipartimento di Biotecnologie Cellulari ed Ematologia, Sezione di Genetica Molecolare, Sapienza., Università di Roma, Roma, Italy. ${ }^{5}$ NIHR-Leeds

Musculoskeletal Biomedical Research Unit (NIHR-LMBRU), Leeds Institute of Rheumatic and Musculoskeletal Medicine, Leeds, UK. ${ }^{6}$ University of Pavia, Pavia, Italy. ${ }^{7}$ Università di Siena, Siena, Italy. ${ }^{8}$ Department of Life Sciences, Università di Siena, Siena, Italy. ${ }^{9}$ Department of Pediatrics, Rheumatology Unit, Anna Meyer Children's Hospital and University of Florence, Florence, Italy. ${ }^{10}$ Department of Medical Sciences, Surgical and Neuroscience. Rheumatology Unit., University of Siena, Siena, Italy.

Published: 8 November 2013

doi:10.1186/1546-0096-11-S1-A187

Cite this article as: Lucherini et al:: OR10-004 - Circulating micrornas in TRAPS. Pediatric Rheumatology 2013 11(Suppl 1):A187. 